\title{
Cancer models for reverse and forward translation
}

\author{
Robust and faithful preclinical models are essential for understanding the underlying biology of human cancer and \\ for devising new and improved therapies.
}

S ometimes to go forward, you must first take a step back. This is also the case in cancer research, as developing new treatments for patient use first requires going back to the lab to understand the underlying tumor biology and to explore new therapeutic avenues, before testing and ultimately adopting the most promising ones in the clinic. This mix of forward and reverse translation of fundamental research and clinical findings, respectively, is the bedrock of modern cancer research and oncology. To be successful, it requires robust cancer models that are faithful - to the extent possible - to the human tumor context under study. This represents an enduring challenge for the field that is being tackled with increasingly greater ingenuity as technology advances.

The addition of new types of in vitro, in vivo and ex vivo models and the refinement of existing ones currently provides investigators with a large roster of systems that can frequently complement and overcome each other's distinct advantages and disadvantages. Among them, patient-derived xenograft (PDX) models, tumor-derived organoids and organotypic cultures from fresh tumor tissue $\mathrm{e}^{1,2}$ are prime examples of sophisticated systems that more faithfully model the biology of human tumors, but not without crucial inherent caveats and limitations. In this issue of Nature Cancer, two papers expand on the value of two distinct types of such cancer models for translational cancer research.

Welm and colleagues present a large biobank of xenograft and matched organoid models derived from patients with breast cancer ${ }^{3}$. This PDX and PDX-derived organoid (PDxO) collection includes valuable models derived from endocrine-resistant, ER+ and HER2+ tumors, treatment-refractory tumors and metastatic tumors, and in some cases represents pairs of primary and metastatic tumors or longitudinally collected samples from the same patient. By enriching existing collections with models representative of some of the deadliest forms of breast cancer, this biobank fills a key gap in this field.

The authors characterized the derived PDXs comprehensively at the genomic, transcriptomic and phenotypic level to establish them as representative of the original patient tumors and provide valuable data recording their heterogeneity. Subsequently generated matched PDxO lines were similarly rigorously analyzed to demonstrate that they retained their original features after long-term culture, as well as the characteristics of their originating tumors and PDXs. A number of PDxOs were used in drug screens showing that their responses recapitulated drug responses of PDXs in vivo, thereby validating their utility as a drug-testing platform. In a proof-of-principle study, the authors further demonstrated the potential of such models to be used as patient avatars to inform treatment decisions by presenting the case of a patient with metastatic triple-negative breast cancer.

The depth and value of this biobank, but also the challenges that remain to be addressed in the use of models of this type, such as the lack of a tumor microenvironment and immune system that recapitulate the human setting, are discussed in detail in the accompanying News \& Views article by Portman and $\mathrm{Lim}^{4}$.

In a separate study, Straussman and colleagues tackled the issues of cancer model fidelity and drug testing by optimizing ex vivo organotypic cultures (EVOCs) of freshly resected human tumors ${ }^{5}$. The authors validated their methodology robustly, including a demonstration that EVOC responses to clinically relevant drugs matched those of established PDX models from colon, lung and breast cancer. They then used this method on freshly resected human colorectal cancer tumors to analyze responses to various drug combinations that were first established by in vitro high-throughput screens. Of the five drug combinations tested in colorectal cancer EVOCs, the authors homed in on the efficacy of combining a MEK and Src inhibitor for a subset of tumors, in particular with the inclusion of standard-of-care chemotherapy. They further identified phosphorylated Src as predictive of MEK and Src inhibition in the pre-treatment setting when KRAS G12 mutations were not present, highlighting the value of such cultures not only for elucidating tumor biology but also for their potential as surrogate systems for predicting patient responses to treatment.

An important advantage of EVOCs over in vitro models, including $\mathrm{PDxOs}$, is that they retain the native microenvironment and architecture of the originating tumor. Conversely, a key limitation is their transient nature, as they cannot be propagated in the manner of PDXs and PDxOs, nor can they be easily preserved. Nevertheless, they can be a powerful addition to the cancer researcher's toolbox, especially when complemented by models that can also address biology and treatment response in vivo.

Together, these studies not only provide valuable in vitro and ex vivo models for fundamental cancer research but also showcase the potential of such systems to be used as forward translation platforms to identify promising treatments and predictive biomarkers that can continue on the path to the clinic.

Published online: 28 February 2022 https://doi.org/10.1038/s43018-022-00346-5

\footnotetext{
References

1. Honkala, A. et al. Nat. Rev. Drug Discov. 21, 99-114 (2022).

2. Lo, Y. H., Karlsson, K. \& Kuo, C. J. Nat. Cancer 1, 761-773 (2020).

3. Guille, K. P. et al. Nat. Cancer https://doi.org/10.1038/s43018-022 00337-6 (2022).

4. Portman, N. \& Lim, E. Nat. Cancer https://doi.org/10.1038/ s43018-021-00328-z (2022).

5. Gavert, N. et al. Nat. Cancer https://doi.org/10.1038/s43018-02100325-2 (2022).
} 